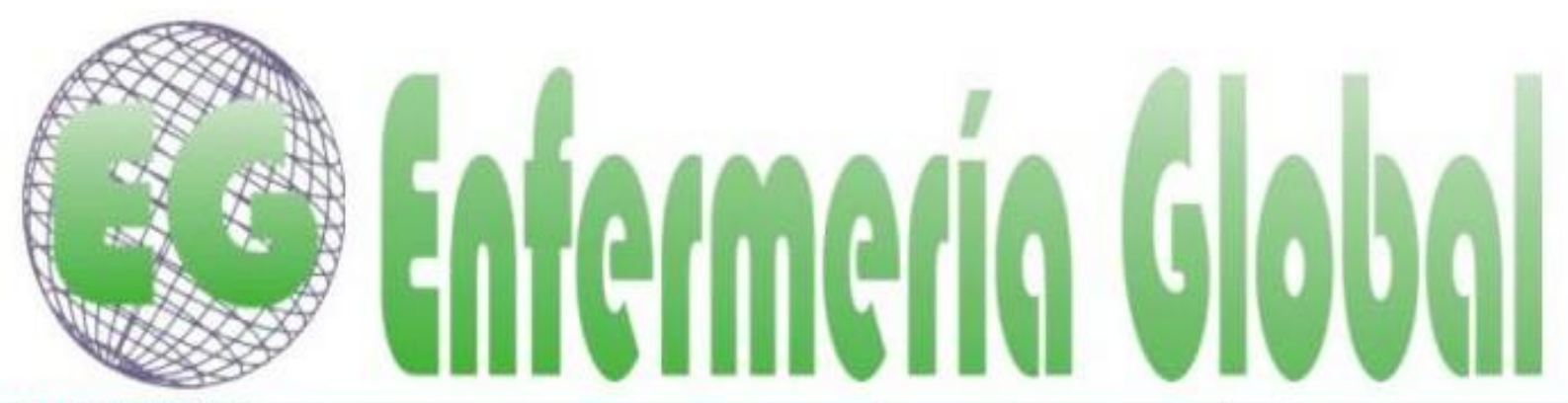

\title{
Instrumento de valoración familiar por el modelo de dominios de la taxonomía II de NANDA
}

Family assessment instrument based on the Nanda taxonomy II domains model

\author{
*Camargo Hernández, Katherine del Consuelo **Pabón Varela, Yadira \\ *Magister en Ciencias en Enfermería, Facultad de Enfermería Universidad Cooperativa de Colombia \\ sede Bucaramanga. E-mail: katherine.camargoh@campusucc.edu.co **Magister en Enfermería, \\ Facultad de Enfermería Universidad Cooperativa de Colombia sede Santa Marta. Colombia.
}

http://dx.doi.org/10.6018/eglobal.16.1.2227951

\section{RESUMEN}

Introducción: Una familia es saludable cuando se logra un equilibrio entre el control, crecimiento, la estabilidad y la espiritualidad de cada uno de sus integrantes con el entorno que los rodea ${ }^{(2)}$.

Objetivo: Diseñar un instrumento validado que permita la valoración familiar por el Modelo de Dominios de la Taxonomía II de NANDA.

Metodología: Diseño cuantitativo, de tipo tecnológica psicométrica con análisis de datos descriptivos. La muestra para la validación del instrumento fue de 8 profesionales en enfermería; además, se realizó una prueba piloto en donde participaron 40 familias pertenecientes a las ciudades de Bucaramanga y Santa Marta, Colombia, 20 para cada ciudad. Para la validación de contenido, se tuvo en cuenta el Modelo de Lawshe Modificado.

Resultados: Existió un consenso general entre los jueces evaluadores en la validación de contenido del Instrumento, el Coeficiente Alpha de Cronbach fue de 0.847 , la correlación no paramétrica Rho de Spearman, arrojó una buena correlación entre los ítems contemplados en el instrumento, con un valor de $r$ mayor de 0.5 y un nivel de significancia $<$ de 0.05 , con un valor de $p$ de cero.

Conclusiones: Se encontró consenso entre los jueces evaluadores, una fiabilidad por medio del Alpha de Cronbach superior a 0.7 y correlaciones no paramétrica Rho de Spearman significativas entre algunos ítems del instrumento.

Palabras clave: Familia; valoración; instrumento; dominios; taxonomía 


\section{ABSTRACT}

Introduction: A family is healthy when a balance among the control, growth, stability and spirituality of each of its members is achieved with the surrounding environment ${ }^{(2)}$.

Objective: To design a validated instrument that permits family assessment based on the North American Nursing Diagnosis Association (NANDA) Taxonomy II domains model.

Methodology: A quantitative design, of the psychometric technological type with descriptive data analysis. The sample for the validation of the instrument comprised eight nursing professionals. In addition, a pilot test was conducted; 40 families from the cities of Bucaramanga and Santa Marta, Colombia, including 20 from each city, participated. For the content validation, the Modified Lawshe's Model was considered.

Results: There was a general consensus among the evaluating judges regarding the validation of the content of the instrument. Cronbach's alpha was 0.847 . Regarding Spearman's rho nonparametric correlation, there was a good correlation among the items considered by the instrument, with a value of $r$ greater than 0.5 , a significance level of $<0.05$ and a $p$-value of zero.

Conclusions: A bibliographical review was performed, according to the context of family. The Family Assessment Instrument, based on the NANDA Taxonomy II Domains Model and consisting of 45 items, was designed. Consensus among the evaluating judges, a reliability by means of Cronbach's alpha value greater than 0.7 and significant Spearman's rho nonparametric correlations among some items of the instrument were found.

Keywords: Family; assessment; instrument; domains; taxonomy

\section{INTRODUCCIÓN}

La familia es considerada en Colombia como "el núcleo fundamental de la sociedad. Se constituye por vínculos naturales o jurídicos, por la decisión libre de un hombre y una mujer de contraer matrimonio o por la voluntad responsable de conformarla." ${ }^{(1)}$. Por tanto es un tema primordial de estudio a lo largo de la historia de la humanidad y en el ejercicio profesional en enfermería.

El objetivo del cuidado enfermero es realizar intervenciones que generen bienestar en la persona, familia o comunidades, dependiendo de las necesidades propias de cada una de ellas. Una familia es saludable cuando se logra un equilibrio entre el control, crecimiento, la estabilidad y la espiritualidad de cada uno de sus integrantes con el entorno que los rodea ${ }^{(2)}$. Para proporcionar un cuidado que responda de forma completa a las necesidades de cada familia, es indispensable contar con herramientas de valoración en enfermería que permitan la identificación de problemas o factores de riesgo, que generen o puedan generar alteraciones en el estado de salud del núcleo familiar.

Existen familias denominadas multiproblemáticas, las cuales son consideradas como "un sistema vulnerable de alto riesgo para todos sus miembros" (3) por tal motivo, es importante diseñar, evaluar y dar a conocer programas que contribuyan efectivamente en detener la disfunción que presentan este tipo de familias. ${ }^{(3)}$ Sin embargo, en enfermería hay pocos instrumentos diseñados para este fin, si la familia es valorada de forma correcta, permitiría al profesional en enfermería realizar intervenciones adecuadas según el estado en el que la familia se encuentre. Los instrumentos de evaluación familiar permiten identificar múltiples factores que mediante el análisis del resultado arrojado se pueda observar en qué medida la familia es funcional o disfuncional, y cuál es el rol que se puede estar interviniendo en el proceso salud- 
enfermedad. Dentro de estos instrumentos se encuentran el apgar familiar, el familiograma, el ecomapa, entre otros, los cuales han sido utilizados por varios años en la labor de enfermería ${ }^{(4)}$. Estas herramientas, proveen un importante aporte en la valoración familiar, pero no están enfocados hacia la Taxonomía II de NANDA.

Lima $R$, Lima $S$, Jimenez $P$ y Domínguez $S$, establecen que la valoración familiar debe tener en cuenta "datos generales de la familia, la composición y estructura, el ciclo vital familiar, el clima social familiar, la integridad familiar, el funcionamiento o dinámica familiar, la resistencia familiar, el afrontamiento familiar y el estudio de los acontecimientos que le afectan" (5). Por tal motivo, se estableció la importancia de crear y validar un instrumento de valoración familiar por el modelo de dominios de la Taxonomía II de NANDA 2012-2014, puesto que en el ejercicio de la profesión, es indispensable tener un referente dentro del lenguaje estandarizado disciplinar que aborde las diversas dimensiones que conforman la familia en el proceso de cuidado.

Algunas etiquetas diagnósticas se pueden utilizar en el proceso de atención de enfermería cuando se interviene la familia como: "disposición para mejorar el afrontamiento familiar, disposición para mejorar los procesos familiares, procesos familiares disfuncionales: alcoholismo, conflicto de decisiones, afrontamiento familiar, deterioro parental, manejo inefectivo del régimen terapéutico familiar, deterioro en el mantenimiento del hogar, entre otros" (6). La mayoría de estas etiquetas diagnósticas, se ven reflejadas en los dominios Promoción de la Salud, Rol/Relaciones, Afrontamiento/Tolerancia al Estrés y Principios Vitales, presentes en la Taxonomía II de NANDA 2012-2014.

Este instrumento busca identificar el estado de salud de la familia valorando los cuatro dominios mencionados anteriormente, haciendo énfasis en tres áreas enunciadas en la Teoría de Sistemas de Betty Neuman, puesto que por medio de ellas, permiten y facilitan al enfermero(a), crear un plan de cuidados de acuerdo a las necesidades propias de cada familia.

\section{OBJETIVOS}

- Diseñar un instrumento validado que permita la valoración familiar por el Modelo de Dominios de la Taxonomía II de NANDA.

- Revisar estudios relacionados con el Modelo de dominios de la taxonomía II de NANDA y el Modelo de Lawshe modificado.

- Diseñar un instrumento para evaluar el estado de salud de la familia basado en el modelo de dominios de la taxonomía II de NANDA 2012-2014.

- Validar el instrumento de valoración familiar basado en el modelo de dominios de la taxonomía II de NANDA 20012-2014 a través de validación de expertos y validación facial o de contenido.

\section{MATERIAL Y MÉTODOS}

Diseño cuantitativo, de tipo tecnológica psicométrica con análisis de datos descriptivos, muestreo no probabilístico por conveniencia. La población para la validación de contenido y validación facial o de apariencia para este instrumento estuvo compuesta por ocho enfermeras con nivel de escolaridad de pregrado, especialización y maestría, que tuvieran conocimiento sobre: proceso de enfermería, de acuerdo a la Taxonomía II de NANDA, instrumentos de valoración familiar y metodología de investigación. Se realizó una prueba piloto en donde participaron 
cuarenta familias pertenecientes a un barrio de la ciudad de Bucaramanga y Santa Marta, Colombia, veinte para cada ciudad, quienes debían residir de manera permanente en el barrio de estas ciudades, debían encontrarse dentro de la vivienda en el momento de responder a las preguntas del instrumento y ser mayores de edad (mayor de 18 años). El proyecto se dividió en dos fases:

Fase I (Revisión de literatura y diseño del instrumento): En esta fase, se realizó revisión bibliográfica acerca de la familia, tipos de familia, salud familiar, funcionalidad familiar, valoración familiar, herramientas para la valoración familiar como familiograma, APGAR familiar, ecomapa; se revisó la teoría de Betty Neuman con algunas de las áreas presentes en la teoría como son: fisiológica, psicológica y sociocultural, además se realizó la revisión de algunos dominios de la Taxonomía II de NANDA 2012-2014, tales como: Promoción de la Salud, Rol/Relaciones, Afrontamiento/Tolerancia al Estrés y Principios Vitales, los cuales fueron base fundamental para el diseño del instrumento.

El Instrumento de Valoración Familiar por el Modelo de Dominios de la Taxonomía II de NANDA diseñado, contiene 45 preguntas distribuidas en los cuatro dominios mencionados anteriormente, los cuales de acuerdo a la literatura encontrada en esta investigación, fueron los predominantes para la valoración familiar, estos dominios se llamaron niveles en el proceso de elaboración del instrumento, además se tuvieron en cuenta tres áreas, para la elaboración del mismo: fisiológica, socio-cultural y psicológica, puesto que se ha evidenciado que existe una relación entre estas áreas con el comportamiento de salud de una familia. Louro $\mathrm{B}$, enunció que existen aspectos psicológicos, sociales, genéticos, ambientales, relacionales y biológicos, que participan en la relación entre la salud y el núcleo familiar, en donde la familia "provee experiencias potenciadoras de salud, asume en forma constructiva y creativa las exigencias que devienen de cada etapa de desarrollo biosicosocial de sus integrantes, y de la vida familiar y social" (7).

Para realizar la puntuación de cada uno de los reactivos o ítems del instrumento, se utilizó la escala de Likert modificada, quedando de la siguiente forma: Nunca: 1 punto, A veces: 2 puntos, Casi siempre: 3 puntos, Siempre: 4 puntos. Cabe resaltar que algunos de los reactivos la puntuación cambió a: Nunca: 4 puntos, A veces: 3 puntos, Casi siempre: 2 puntos, Siempre: 1 punto; esto se debe a que estos reactivos hacen referencias conductas no saludables. Además por sugerencia de uno de los jueces evaluadores, se incorporó una casilla llamada No aplica: cuyo valor es de 0 (cero), puesto que puede que algunos de los ítems no sean aplicables en las familias a intervenir. Al realizar la prueba piloto en la población objeto, se recolectaron datos socio-demográficos, el cual contenía 21 preguntas de tipo dicotómicas y de selección múltiple.

Fase II (Fase de validación del instrumento): Durante el proceso de validación de contenido se tuvo como referencia el modelo de Lawshe Modificado que permite evaluar la validez de contenido. Este modelo permite un juicio de expertos de número reducido, que exige valores mínimos para la aceptación de los ítems en general, sobre todo si existen pocos expertos lo cual es aceptable en investigación; además este modelo, inicia en base al modelo planteado por Lawshe, el cual hace referencia a que un grupo de expertos deben evaluar el contenido de una prueba o un grupo de reactivos, en donde deben emitir un juicio en tres categorías: esencial, útil pero no esencial, no necesario ${ }^{(8)}$. 
Tristán L, enunció que después de obtener esta información se procede a revisar el número de coincidencias en las respuestas, con lo cual se dice que para que el instrumento tenga validez los expertos deben haber coincidido con el cincuenta (50\%) como mínimo en la casilla de categoría esencial. Para realizar el cálculo del acuerdo entre los panelistas en esta categoría (esencial), de acuerdo al Modelo de Lawshe Modificado, se debe realizar el Cálculo de la Razón de Validez de Contenido (CVR') por sus siglas en inglés. Después de calcular la CVR' se procede a calcular el Índice de Validez de Contenido (CVI) por sus siglas en inglés, lo cual indica el promedio de los reactivos aceptables.

Además este autor enunció que:

Para fines prácticos se puede decir que CVR' debe ser proporcionar por lo menos un $58 \%$ para ser aceptable, este valor es constante, independientemente del número de panelistas, con lo cual no solamente se resuelve el problema del efecto de tamaño, sino también la interpretación de los acuerdos en la relación de validez de contenido. Como CVR' no depende de $\mathrm{N}$, cuando $\mathrm{N}$ tiene a, CVR' permanece constante en 0.5823 , lo cual conduce a una condición de igual exigencia en todos los casos, independientemente del número de panelistas, lo cual parece mucho más realista que lo establecido en el modelo de CVR propuesto por Lawshe.

El responsable del diseño del instrumento puede convocar a los miembros del panel de validez de contenido para hacer una revisión en los casos en los cuales no se alcanzó el mínimo del 58\% del consenso en la categoría "esencial", esta revisión permitirá rescatar algunos de los ítems donde no hubo acuerdo o, en su caso, sugerir modificaciones para futuras revisiones. Por tratarse el CVI del promedio de los ítems aceptables de acuerdo con el CVR', se espera que brinde valores superiores a 0.58, de ahí surge una aplicación interesante para dictaminar la validez de un instrumento o de un banco de ítems y que se puede plantear como una extensión del modelo de Lawshe ${ }^{(9)}$.

Al terminar el proceso de recolección de la información se procedió a tabular y organizar la información para realizar las correcciones pertinentes al instrumento, para lo cual se contó con la asesoría de un profesional en estadística quien contribuyó en el proceso de organización de la información y la realización del análisis de la información acorde al modelo de Lawshe Modificado. Se realizó la validez facial o de apariencia con el fin de comprobar la comprensión y claridad de cada ítem, que fue también revisada por parte del panel de expertos a los cuales se entregaba un formato que permitía la evaluación de cada ítem del instrumento a validar, permitiendo escribir las respectivas observaciones y recomendaciones, para mejorar el instrumento y hacer las correcciones propuestas.

Para Sánchez $R$ y Echeverry J, en la validez facial o de apariencia "la escala parece medir lo que debe medir" (10) y Díaz L, Muñoz A y De Vargas D, establecieron que en esta validación, se debe tener como referencia "la apreciación subjetiva de personas expertas y de otras que cuenten con las mismas características de los posibles usuarios; y, mediante los criterios de claridad, precisión y comprensión, se determinó la validez facial."(11)

Luego de realizar las correcciones pertinentes se realizó una prueba piloto a veinte familias pertenecientes a un barrio de la ciudad de Bucaramanga, Santander, 
Colombia y veinte familias pertenecientes a un barrio de la ciudad de Santa Marta, Magdalena, Colombia, elegidas por muestreo no probabilístico por conveniencia, quienes aceptaron la participación en la investigación. Previa a la aceptación por parte de las familias en la participación del proyecto de investigación, se realizó la explicación, acerca del propósito del proyecto de investigación, por medio de una comunicación directa y de la información contemplada en la entrega del consentimiento informado. Posteriormente a esto, se inició la recolección de los datos y la puesta en marcha de la prueba piloto en estas familias.

Finalmente se procedió a tabular la información obtenida y evaluar los resultados de la prueba piloto para verificar la aplicabilidad de cada uno de los ítems del instrumento de valoración familia.

\section{RESULTADOS}

Tabla 1. Resultados del Cálculo de la Razón de Validez de Contenido (CVR) con el Modelo de Lawshe Modificado.

\begin{tabular}{|c|c|c|c|c|c|}
\hline $\begin{array}{c}N \\
\text { (\#Panelistas) }\end{array}$ & Ítems & Esencial & $\begin{array}{c}\text { Útil pero } \\
\text { no } \\
\text { Esencial }\end{array}$ & $\begin{array}{c}\text { No } \\
\text { Necesario }\end{array}$ & CVR' \\
\hline 8 & 1 & 7 & 1 & 0 & 0,88 \\
\hline 8 & 2 & 8 & 0 & 0 & 1,00 \\
\hline 8 & 3 & 8 & 0 & 0 & 1,00 \\
\hline 8 & 4 & 6 & 1 & 1 & 0,75 \\
\hline 8 & 5 & 8 & 0 & 0 & 1,00 \\
\hline 8 & 6 & 4 & 3 & 1 & 0,50 \\
\hline 8 & 7 & 7 & 1 & 0 & 0,88 \\
\hline 8 & 8 & 7 & 1 & 0 & 0,88 \\
\hline 8 & 9 & 6 & 1 & 1 & 0,75 \\
\hline 8 & 10 & 8 & 0 & 0 & 1,00 \\
\hline 8 & 11 & 4 & 4 & 0 & 0,50 \\
\hline 8 & 12 & 5 & 3 & 0 & 0,63 \\
\hline 8 & 13 & 7 & 1 & 0 & 0,88 \\
\hline 8 & 14 & 8 & 0 & 0 & 1,00 \\
\hline 8 & 15 & 8 & 0 & 0 & 1,00 \\
\hline 8 & 16 & 8 & 1 & 0 & 0,88 \\
\hline 8 & 17 & 7 & 0 & 1 & 0,88 \\
\hline 8 & 18 & 7 & 1 & 0 & 0,88 \\
\hline 8 & 19 & 7 & 1 & 0 & 0,88 \\
\hline 8 & 20 & 7 & 1 & 0 & 0,88 \\
\hline 8 & 21 & 7 & 1 & 0 & 0,88 \\
\hline 8 & 22 & 7 & 1 & 0 & 0,88 \\
\hline 8 & 23 & 7 & 1 & 0 & 0,88 \\
\hline 8 & $24^{*}$ & 5 & 2 & 0 & 0,63 \\
\hline 8 & 25 & 5 & 3 & 0 & 0,63 \\
\hline 8 & 26 & 7 & 1 & 0 & 0,88 \\
\hline 8 & 27 & 6 & 2 & 0 & 0,75 \\
\hline 8 & 28 & 7 & 1 & 0 & 0,88 \\
\hline 8 & 29 & 8 & 0 & 0 & 1,00 \\
\hline 8 & 30 & 6 & 1 & 1 & 0,75 \\
\hline 8 & 31 & 6 & 2 & 0 & 0,75 \\
\hline & & & & & \\
\hline
\end{tabular}




\begin{tabular}{|l|l|l|l|l|l|}
\hline 8 & 32 & 8 & 0 & 0 & 1,00 \\
\hline 8 & 33 & 8 & 0 & 0 & 1,00 \\
\hline 8 & 34 & 6 & 2 & 0 & 0,75 \\
\hline 8 & 35 & 8 & 0 & 0 & 1,00 \\
\hline 8 & 36 & 5 & 1 & 2 & 0,63 \\
\hline 8 & 37 & 7 & 1 & 0 & 0,88 \\
\hline 8 & 38 & 6 & 0 & 2 & 0,75 \\
\hline 8 & 39 & 7 & 1 & 0 & 0,88 \\
\hline 8 & 40 & 8 & 0 & 0 & 1,00 \\
\hline 8 & 41 & 8 & 0 & 0 & 1,00 \\
\hline 8 & 42 & 8 & 0 & 0 & 1,00 \\
\hline 8 & 43 & 6 & 2 & 0 & 0,75 \\
\hline 8 & 44 & 5 & 1 & 2 & 0,63 \\
\hline 8 & 45 & 7 & 1 & 0 & 0,88 \\
\hline
\end{tabular}

* Un Juez no contestó porque no entendió el reactivo

Fuente: Instrumento de Valoración Familiar

Al establecer el análisis de los datos obtenidos con la validación de expertos, en el Instrumento de Valoración Familiar por el Modelo de Dominios de la Taxonomía II de NANDA, con el Modelo de Lawshe Modificado se puede determinar que 43 de los reactivos establecidos en el instrumento, poseen una validez de contenido, logrando un consenso general en la mayoría de los jueces, sólo los ítems número 6 y 11 no tuvieron un CVR' mayor de 0,58.

Tabla 2. Resultados del Índice de Validez de Contenido (CVI) con el Modelo de Lawshe Modificado.

\begin{tabular}{|c|c|}
\hline Items & CVR' \\
\hline 1 & 0,88 \\
\hline 2 & 1,00 \\
\hline 3 & 1,00 \\
\hline 4 & 0,75 \\
\hline 5 & 1,00 \\
\hline 6 & 0,50 \\
\hline 7 & 0,88 \\
\hline 8 & 0,88 \\
\hline 9 & 0,75 \\
\hline 10 & 1,00 \\
\hline 11 & 0,50 \\
\hline 12 & 0,63 \\
\hline 13 & 0,88 \\
\hline 14 & 1,00 \\
\hline 15 & 1,00 \\
\hline 16 & 0,88 \\
\hline 17 & 0,88 \\
\hline 18 & 0,88 \\
\hline 19 & 0,88 \\
\hline 20 & 0,88 \\
\hline 21 & 0,88 \\
\hline 22 & 0,88 \\
\hline 23 & 0,88 \\
\hline $24^{*}$ & 0,63 \\
\hline & \\
\hline
\end{tabular}




\begin{tabular}{|c|c|}
\hline 25 & 0,63 \\
\hline 26 & 0,88 \\
\hline 27 & 0,75 \\
\hline 28 & 0,88 \\
\hline 29 & 1,00 \\
\hline 30 & 0,75 \\
\hline 31 & 0,75 \\
\hline 32 & 1,00 \\
\hline 33 & 1,00 \\
\hline 34 & 0,75 \\
\hline 35 & 1,00 \\
\hline 36 & 0,63 \\
\hline 37 & 0,88 \\
\hline 38 & 0,75 \\
\hline 39 & 0,88 \\
\hline 40 & 1,00 \\
\hline 41 & 1,00 \\
\hline 42 & 1,00 \\
\hline 43 & 0,75 \\
\hline 44 & 0,63 \\
\hline 45 & 0,88 \\
\hline \multicolumn{2}{|c|}{} \\
\hline SUMA TODOS LOS ITEMS & 38,00 \\
\hline SUMA ITEMS ACEPTABLES & 37,00 \\
\hline * Un Juez no contestó porque no entendió el reactivo \\
Fuente: Instrumento de Valoración Familiar \\
\hline
\end{tabular}

Al realizar el CVR' en el Instrumento de Valoración Familiar por el Modelo de Dominios de la Taxonomía II de NANDA, sólo los reactivos número 6 (Algún miembro de su familia se encuentra apático, cansado o sin motivación frente a las actividades recreativas) y 11 (En su familiar los menores de edad piden autorización para realizar actividades fuera de la casa), no cumplieron con la constante de CVR' (Razón de Validez de Contenido por el Modelo de Lawshe Modificado) mayor de 0,58, para lo cual, se realizó nuevamente la revisión de los datos obtenidos por cada uno de los jueces y se analizó las observaciones que cada uno de ellos estableció para cada reactivo obteniendo como resultado, una modificación de los mismos.

\section{Validez Facial o de Apariencia}

Dentro de los estándares establecidos en la segunda fase de esta investigación, se determinó que la validez facial o de apariencia se iba a realizar mediante una prueba piloto, en donde se conformaron dos grupos, uno de sujetos que van a ser medidos con la escala y otro de expertos, éstos últimos analizan la escala y establecen si el instrumento mide o no lo que se propone. Por tanto, antes de aplicar el instrumento de Valoración Familiar por el Modelo de Dominios de la Taxonomía II de NANDA diseñado en la primera fase, se tuvieron en cuenta las observaciones realizadas por los jueces evaluadores con respecto a la apariencia, orden y redacción de cada uno de los 45 ítems establecidos en el instrumento diseñado.

Al realizar la aplicabilidad del instrumento mediante la prueba piloto a dos comunidades pertenecientes a las ciudades de Bucaramanga y Santa Marta, Colombia, no se encontraron observaciones con respecto a los ítems, es decir, cada 
uno de ellos estaban enunciados en una forma clara, sencilla y entendible para estas comunidades, según refirieron los participantes en esta prueba.

Una vez tabulados los datos obtenidos de la prueba piloto se realizó la fiabilidad del Instrumento, por medio del coeficiente Alpha de Cronbach, el cual arrojó un resultado de 0.847 .

\section{Correlación no paramétrica Rho de Spearman}

Proseguido a la realización de la fiabilidad del instrumento, se llevó a cabo la correlación de cada uno de los ítems entre sí, para ello se realizó la correlación paramétrica Rho de Spearman puesto que las variables de este instrumento son ordinales, no numéricas ni tampoco nominales ${ }^{(12)}$.

En la tabla 3, se encuentra el reactivo número 7 el cual pertenece al dominio promoción de la salud y los reactivos 12, 19, 20, 21 y 23, pertenecen al dominio Rol/relaciones, indicando que entre estos dos dominios existe una correlación significativa, lo cual se puede evidenciar en lo enunciado por Giraldo O, Toro R, Macías L, Valencia G y Palacio R, cuando establecen que la Promoción de la Salud, de acuerdo al modelo teórico de Nola Pender, tiene relación a los estilos de vida saludables, en donde "los factores cognitivos-perceptuales de los individuos, son modificados por las condiciones situacionales, personales e interpersonales, para lo cual la cultura es tenida en cuenta en razón a la forma que influye en la toma de decisiones de las personas" (13).

Tabla 3. Correlaciones no Paramétricas Rho de Spearman entre el Ítem 7 con otros ítems.

\begin{tabular}{|c|c|c|c|}
\hline Ítem & Ítems & $\begin{array}{l}\text { Valor } \\
\text { de } r\end{array}$ & $\begin{array}{l}\text { Valor } \\
\text { de } p\end{array}$ \\
\hline \multirow{5}{*}{$\begin{array}{l}\text { 7. Su familia acude } \\
\text { a consulta médica o } \\
\text { a los programas de } \\
\text { detección temprana } \\
\text { y protección } \\
\text { específica. }\end{array}$} & $\begin{array}{l}\text { 12. En su familia se realiza control } \\
\text { disciplinario y se aplican sanciones a los } \\
\text { menores de edad. }\end{array}$ & 0,568 & 0 \\
\hline & $\begin{array}{l}\text { 19. La vida social de su familia, se ha visto } \\
\text { afectada a cusa del cuidado brindado a la } \\
\text { persona dependiente (limitación física y } \\
\text { mental). }\end{array}$ & 0,699 & 0 \\
\hline & $\begin{array}{l}\text { 20. Al brindar cuidado a la persona } \\
\text { dependiente (limitación física y mental), se ha } \\
\text { perdido la unión familiar. }\end{array}$ & 0,692 & 0 \\
\hline & $\begin{array}{l}\text { 21. En su familia a causa de brindar cuidados } \\
\text { a la persona dependiente (limitación física y } \\
\text { mental), se ha presentado algún sentimiento } \\
\text { negativo como rabia, impotencia, intolerancia. }\end{array}$ & 0,687 & 0 \\
\hline & $\begin{array}{l}\text { 23. En su familia, existe alguna alteración en } \\
\text { la salud de algún integrante, a causa de } \\
\text { brindar cuidado a la persona dependiente } \\
\text { (limitación física y mental) }\end{array}$ & 0,580 & 0 \\
\hline
\end{tabular}

Fuente: Instrumento de Valoración Familiar

Los ítems contemplados en la tabla 4 y 5 , pertenecen al dominio Rol/relaciones, dominio que es muy importante en el momento de realizar valoración familiar, puesto que el ambiente en el que se rodee el ser humano, influye de forma positiva 0 
negativa en su salud física y mental. Los aspectos biológicos, económicos, educativos, espirituales, tiene una marcada importancia en la familia, puesto que por medio de ella "se desarrollan valores, creencias, conocimientos, criterios, juicios que determinan la salud de los individuos y del colectivo de sus integrantes" (14). Además, en la valoración de este dominio es importante revisar las conexiones y asociaciones tanto negativas como positivas entre todos los miembros del núcleo familiar o grupos de personas y los medios por los cuales se demuestran tales conexiones, esto a fin de valorar el cumplimiento de los roles dentro de la familia y al mismo tiempo definir las posibles intervenciones necesarias para corregir conductas inadecuadas ${ }^{(15)}$.

Tabla 4. Correlaciones no Paramétricas Rho de Spearman entre el ítem 11 con otros ítems.

\begin{tabular}{|l|l|c|c|}
\hline \multicolumn{1}{|c|}{ Ítem } & \multicolumn{1}{|c|}{ Ítems } & $\begin{array}{c}\text { Valor } \\
\text { de } r\end{array}$ & $\begin{array}{l}\text { Valor } \\
\text { de } p\end{array}$ \\
\hline $\begin{array}{l}\text { 11. En su familia los } \\
\text { menores de edad } \\
\text { piden autorización } \\
\text { para realizar } \\
\text { actividades fuera de } \\
\text { la casa. }\end{array}$ & $\begin{array}{l}\text { 13. En su familia los menores de edad } \\
\text { comunican a los adultos sus problemas y } \\
\text { necesidades. }\end{array}$ & 0,626 & 0,00 \\
\hline
\end{tabular}

Fuente: Instrumento de Valoración Familiar

Tabla 5. Correlaciones no Paramétricas Rho de Spearman entre el ítem 13 con otros ítems.

\begin{tabular}{|c|c|c|c|}
\hline Ítem & Ítems & $\begin{array}{l}\text { Valor } \\
\text { de } r\end{array}$ & $\begin{array}{l}\text { Valor } \\
\text { de } p\end{array}$ \\
\hline \multirow{4}{*}{$\begin{array}{l}\text { 13. En su familia los } \\
\text { menores de edad } \\
\text { comunican a los } \\
\text { adultos sus } \\
\text { problemas y } \\
\text { necesidades. }\end{array}$} & $\begin{array}{l}\text { 19. La vida social de su familia, se ha visto } \\
\text { afectada a causa del cuidado brindado a la } \\
\text { persona dependiente (limitación física y } \\
\text { mental). }\end{array}$ & 0,555 & 0 \\
\hline & $\begin{array}{l}\text { 20. Al brindar cuidado a la persona } \\
\text { dependiente (limitación física y mental), se ha } \\
\text { perdido la unión familiar). }\end{array}$ & 0,567 & 0 \\
\hline & $\begin{array}{l}\text { 21. En su familia a causa de brindar cuidados } \\
\text { a la persona dependiente (limitación física y } \\
\text { mental), se ha presentado algún sentimiento } \\
\text { negativo como rabia, impotencia, intolerancia. }\end{array}$ & 0,542 & 0 \\
\hline & $\begin{array}{l}\text { 23. En su familia, existe alguna alteración en } \\
\text { la salud de algún integrante, a causa de } \\
\text { brindar cuidado a la persona dependiente } \\
\text { (limitación física y mental). }\end{array}$ & 0,571 & 0 \\
\hline
\end{tabular}

Fuente: Instrumento de Valoración Familiar

En la tabla 6 y 7, se encuentran los ítems 15 y 17, los cuales pertenecen al dominio Rol/relaciones y el 41, al dominio principios vitales, lo que indica que existen correlación entre estos dos dominios y según lo enunciado por Villalobos $\mathrm{V}$, algunas de las familias intervenidas en su estudio, experimentaron dificultades en: "las formas de participación y con lo cual se afecta el sentido de pertenencia al grupo, en la comunicación entre los miembros de la familia" ${ }^{(2)}$, lo que conlleva a pensar que de 
acuerdo a la formación, cultura, valores, creencias, principios, de cada familia, se puede generan conductas positivas o negativas en la salud física y psicológica de los miembros que la conforman.

Tabla 6. Correlaciones no Paramétricas Rho de Spearman entre el ítem 15 con otro ítem.

\begin{tabular}{|l|l|c|c|}
\hline \multicolumn{1}{|c|}{ Ítem } & \multicolumn{1}{|c|}{ Ítems } & $\begin{array}{c}\text { Valor } \\
\text { de } r\end{array}$ & $\begin{array}{c}\text { Valor } \\
\text { de } p\end{array}$ \\
\hline $\begin{array}{l}\text { 15. En su familia cuando } \\
\text { uno de sus integrantes } \\
\text { tiene problemas se le } \\
\text { brinda apoyo. }\end{array}$ & $\begin{array}{l}\text { 41. En su familia tiene deseos de } \\
\text { mejorar la capacidad de afrontamiento, } \\
\text { esperanza, alegría y significado por la } \\
\text { vida ante una dificultad. }\end{array}$ & 0,547 & 0 \\
\hline
\end{tabular}

Fuente: Instrumento de Valoración Familiar

Tabla 7. Correlaciones no Paramétricas Rho de Spearman entre el ítem 17con otro ítem.

\begin{tabular}{|l|l|c|c|}
\hline \multicolumn{1}{|c|}{ Ítem } & \multicolumn{1}{|c|}{ Ítems } & $\begin{array}{c}\text { Valor } \\
\text { de } r\end{array}$ & $\begin{array}{c}\text { Valor } \\
\text { de } p\end{array}$ \\
\hline $\begin{array}{l}\text { 17. En su familia utilizan la } \\
\text { autoridad como método } \\
\text { correctivo (castigo o } \\
\text { sanción frente a una falta } \\
\text { o comportamiento } \\
\text { inadecuado por parte de } \\
\text { algún integrante de la } \\
\text { familia). }\end{array}$ & $\begin{array}{l}\text { 41. En su familia tiene deseos de } \\
\text { mejorar la capacidad de afrontamiento, } \\
\text { esperanza, alegría y significado por la } \\
\text { vida ante una dificultad. }\end{array}$ & 0,541 & 0 \\
\hline
\end{tabular}

Fuente: Instrumento de Valoración Familiar

En las tablas 8, 9 y 10, se observan los ítems número 19, 20 y 21, los cuales pertenecen al dominio Rol/relaciones, en los resultados obtenidos en la investigación, se encontró que aparte de tener correlación con los ítems del mismo dominio (ítems 20, 21, 22 y 23), también tiene relación con el ítem número 32, perteneciente al dominio Afrontamiento/Tolerancia al estrés, esto se ve evidenciado en la revisión de literatura realizada, en donde Gómez $E$ y Kotlierenco $M$, quisieron profundizar en el concepto de resiliencia familiar, como componente útil en la "intervención psicosocial, clínica y de salud con familias altamente vulnerables o multiproblemáticas", ${ }^{(16)}$ estos autores establecieron que en este concepto intervienen unos factores llamados protectores, los cuales intervienen significativamente en el funcionamiento de las familias, para conservarla de forma saludable y prepararla en situaciones estresantes, tal es el caso de las tradiciones, rutinas, celebraciones con los miembros de la familia. $^{(16)}$ 
Tabla 8. Correlaciones no Paramétricas Rho de Spearman entre el ítem 19 con otros ítems.

\begin{tabular}{|c|c|c|c|}
\hline Reactivo & Reactivos & $\begin{array}{l}\text { Valor } \\
\text { de } r\end{array}$ & $\begin{array}{l}\text { Valor } \\
\text { de } p\end{array}$ \\
\hline \multirow{5}{*}{$\begin{array}{l}\text { 19. La vida social } \\
\text { de su familia, se ha } \\
\text { visto afectada a } \\
\text { causa del cuidado } \\
\text { brindado a la } \\
\text { persona } \\
\text { dependiente } \\
\text { (limitación física y } \\
\text { mental). }\end{array}$} & $\begin{array}{l}\text { 20. Al brindar cuidado a la persona } \\
\text { dependiente (limitación física y mental), se ha } \\
\text { perdido la unión familiar. }\end{array}$ & 0,954 & 0 \\
\hline & $\begin{array}{l}\text { 21. En su familia a causa de brindar cuidados } \\
\text { a la persona dependiente (limitación física y } \\
\text { mental), se ha presentado algún sentimiento } \\
\text { negativo como rabia, impotencia, intolerancia. }\end{array}$ & 0,942 & 0 \\
\hline & $\begin{array}{l}\text { 22. En su familia predomina la armonía } \\
\text { (entendimiento, diálogo entre los integrantes } \\
\text { del hogar). }\end{array}$ & 0,568 & 0 \\
\hline & $\begin{array}{l}\text { 23. En su familia, existe alguna alteración en } \\
\text { la salud de algún integrante, a causa de } \\
\text { brindar cuidado a la persona dependiente } \\
\text { (limitación física y mental). }\end{array}$ & 0,815 & 0 \\
\hline & $\begin{array}{l}\text { 32. Su familia tiene la capacidad de superar } \\
\text { momentos difíciles. }\end{array}$ & 0,568 & 0 \\
\hline
\end{tabular}

Fuente: Instrumento de Valoración Familiar

Tabla 9. Correlaciones no Paramétricas Rho de Spearman entre el ítem 20 con otros ítems.

\begin{tabular}{|c|c|c|c|}
\hline Ítem & Ítems & $\begin{array}{l}\text { Valor } \\
\text { de } r\end{array}$ & $\begin{array}{l}\text { Valor } \\
\text { de } p\end{array}$ \\
\hline \multirow{3}{*}{$\begin{array}{l}\text { 20. Al brindar } \\
\text { cuidado a la } \\
\text { persona } \\
\text { dependiente } \\
\text { (limitación física y } \\
\text { mental), se ha } \\
\text { perdido la unión } \\
\text { familiar. }\end{array}$} & $\begin{array}{l}\text { 21. En su familia a causa de brindar cuidados } \\
\text { a la persona dependiente (limitación física y } \\
\text { mental), se ha presentado algún sentimiento } \\
\text { negativo como rabia, impotencia, intolerancia. }\end{array}$ & 0,965 & 0 \\
\hline & $\begin{array}{l}\text { 23. En su familia, existe alguna alteración en } \\
\text { la salud de algún integrante, a causa de } \\
\text { brindar cuidado a la persona dependiente } \\
\text { (limitación física y mental). }\end{array}$ & 0,870 & 0 \\
\hline & $\begin{array}{l}\text { 32. Su familia tiene la capacidad de superar } \\
\text { momentos difíciles. }\end{array}$ & 0,552 & 0 \\
\hline
\end{tabular}

Fuente: Instrumento de Valoración Familiar

Tabla 10. Correlaciones no Paramétricas Rho de Spearman entre el ítem 21 con otros ítems.

\begin{tabular}{|l|l|c|c|}
\hline \multicolumn{1}{|c|}{ Ítem } & \multicolumn{1}{|c|}{ Ítems } & $\begin{array}{c}\text { Valor } \\
\text { de } r\end{array}$ & $\begin{array}{c}\text { Valor } \\
\text { de } p\end{array}$ \\
\hline $\begin{array}{l}\text { 21. En su familia a } \\
\text { causa de brindar } \\
\text { cuidados a la } \\
\text { persona } \\
\text { dependiente } \\
\text { (limitación física y } \\
\text { mental), se ha } \\
\text { presentado algún } \\
\text { sentimiento }\end{array}$ & $\begin{array}{l}\text { 23. En su familia, existe alguna alteración en } \\
\text { la salud de algún integrante, a causa de } \\
\text { brindar cuidado a la persona dependiente } \\
\text { (limitación física y mental). }\end{array}$ & 0,875 & 0 \\
\cline { 2 - 4 } & $\begin{array}{l}\text { 32. Su familia tiene la capacidad de superar } \\
\text { momentos difíciles. }\end{array}$ & 0,547 & 0 \\
\hline
\end{tabular}


negativo como

rabia, impotencia,

intolerancia.

Fuente: Instrumento de Valoración Familiar

En las tablas 11, 12, 13 y 14, está plasmado el ítem: 22, el cual pertenece al dominio Rol/relaciones, los ítems: 26, 27, 28, 29, 31, pertenecen al rol Afrontamiento y Tolerancia al estrés y el reactivo 45 al dominio principios vitales. Estos hallazgos coinciden con lo descrito anteriormente, por Raile A y Marriner T y Lima J, Lima M y Sáez $A$, en donde establecen la relación que existe entre los mismos para la valoración familiar ${ }^{(6),(17)}$.

Tabla 11. Correlaciones no Paramétricas Rho de Spearman entre el ítem 22 con otros ítems.

\begin{tabular}{|c|c|c|c|}
\hline Ítem & Ítems & $\begin{array}{l}\text { Valor } \\
\text { de } r\end{array}$ & $\begin{array}{l}\text { Valor } \\
\text { de } p\end{array}$ \\
\hline \multirow{3}{*}{$\begin{array}{l}\text { 22. En su familia } \\
\text { predomina la } \\
\text { armonía } \\
\text { (entendimiento, } \\
\text { diálogo entre los } \\
\text { integrantes del } \\
\text { hogar). }\end{array}$} & $\begin{array}{l}\text { 26. Se busca soluciones ante los conflictos } \\
\text { militares. }\end{array}$ & 0,611 & 0 \\
\hline & $\begin{array}{l}\text { 27. Se tiene presente las inquietudes de los } \\
\text { miembros de la familia. }\end{array}$ & 0,574 & 0 \\
\hline & $\begin{array}{l}\text { 45. Su familia cumple con todas las } \\
\text { indicaciones médicas para mejorar su estado } \\
\text { de salud. }\end{array}$ & 0,531 & 0 \\
\hline
\end{tabular}

Fuente: Instrumento de Valoración Familiar

Tabla 12. Correlaciones no Paramétricas Rho de Spearman entre el ítem 26 con otro ítem.

\begin{tabular}{|l|l|c|c|}
\hline \multicolumn{1}{|c|}{ Ítem } & \multicolumn{1}{|c|}{ İtems } & $\begin{array}{c}\text { Valor } \\
\text { de } r\end{array}$ & $\begin{array}{c}\text { Valor } \\
\text { de } p\end{array}$ \\
\hline $\begin{array}{l}\text { 26. Se busca } \\
\text { soluciones ante los } \\
\text { conflictos militares. }\end{array}$ & $\begin{array}{l}\text { 27. Se tiene presente las inquietudes de los } \\
\text { miembros de la familia. }\end{array}$ & 0,750 & 0 \\
\hline
\end{tabular}

Fuente: Instrumento de Valoración Familiar

Tabla 13. Correlaciones no Paramétricas Rho de Spearman entre el ítem 27 con otros ítems.

\begin{tabular}{|c|c|c|c|}
\hline Ittem & Ítems & $\begin{array}{l}\text { Valor } \\
\text { de } r\end{array}$ & $\begin{array}{l}\text { Valor } \\
\text { de } p\end{array}$ \\
\hline \multirow{3}{*}{$\begin{array}{l}\text { 27. Se tiene } \\
\text { presente las } \\
\text { inquietudes de los } \\
\text { miembros de la } \\
\text { familia. }\end{array}$} & $\begin{array}{l}\text { 28. Algún integrante de su familia, } \\
\text { proporciona apoyo, ayuda, comprensión, } \\
\text { para superar las crisis. }\end{array}$ & 0,695 & 0 \\
\hline & $\begin{array}{l}\text { 29. Los integrantes de su familia demuestran } \\
\text { respuestas positivas ante una situación difícil. }\end{array}$ & 0,585 & 0 \\
\hline & $\begin{array}{l}\text { 31. En su familia los padres animan a sus } \\
\text { hijos a realizar actividades que demanden } \\
\text { dificultad. }\end{array}$ & 0,581 & 0 \\
\hline
\end{tabular}

Fuente: Instrumento de Valoración Familiar 
Tabla 14. Correlaciones no Paramétricas Rho de Spearman entre el ítem 28 con otro ítem.

\begin{tabular}{|l|l|c|c|}
\hline \multicolumn{1}{|c|}{ Ítem } & \multicolumn{1}{|c|}{ Ítems } & $\begin{array}{c}\text { Valor } \\
\text { de } r\end{array}$ & $\begin{array}{c}\text { Valor } \\
\text { de } p\end{array}$ \\
\hline $\begin{array}{l}\text { 28. Algún integrante } \\
\text { de su familia, } \\
\text { proporciona apoyo, } \\
\text { ayuda, } \\
\text { comprensión, para } \\
\text { superar las crisis. }\end{array}$ & $\begin{array}{l}\text { 29. Los integrantes de su familia demuestran } \\
\text { respuestas positivas ante una situación difícil. }\end{array}$ & 0,686 & 0 \\
\hline
\end{tabular}

Fuente: Instrumento de Valoración Familiar

En la tabla 15, los ítems 40 y 42 , pertenecen al dominio principios vitales, dominios importantes en el momento de realizar valoración familiar, puesto que la cultura es "parte importante de la identidad de cada persona y que es necesario considerar los aspectos culturales que influyen en el cuidado de la salud para responder a una necesidad real de enfermería: cuidar a personas de culturas diferentes que piensan y actúan de forma particularmente especial" (18) además, de acuerdo a la teoría de la diversidad y de la universalidad de los cuidados culturales de Madeline Leinner, la cultura "hace referencia a los valores, creencias, normas, símbolos, prácticas y modos de vida de individuos, grupos o instituciones, aprendidos, compartidos y transmitidos de una generación a otra" ${ }^{17)}$ por tanto ante cualquier intervención terapéutica se hace primordial indagar y conocer las creencias existentes en la familia, para poder lograr el/los objetivo/s terapéutico/s planeado/s en el Proceso de atención de enfermería.

Tabla 15. Correlaciones no Paramétricas Rho de Spearman entre el ítem 40con otro ítem.

\begin{tabular}{|l|l|c|c|}
\hline \multicolumn{1}{|c|}{ Ítem } & \multicolumn{1}{|c|}{ Ítems } & $\begin{array}{c}\text { Valor } \\
\text { de } r\end{array}$ & $\begin{array}{c}\text { Valor } \\
\text { de } p\end{array}$ \\
\hline $\begin{array}{l}\text { 40. En su familia } \\
\text { necesitan creer en } \\
\text { un ser supremo } \\
\begin{array}{l}\text { para llevar a cabo } \\
\text { las actividades } \\
\text { diarias. }\end{array}\end{array}$ & $\begin{array}{l}\text { 42. Su familia participa en actividades } \\
\text { religiosas. }\end{array}$ & 0,563 & 0 \\
\hline
\end{tabular}

Fuente: Instrumento de Valoración Familiar

\section{DISCUSIÓN}

El proceso de atención de enfermería, "tiene como objetivo dar respuesta a las necesidades de la persona, la familia y las comunidades, por tal motivo se hace necesario el tener un instrumento propio de valoración valido" ${ }^{(19)}$, existen algunos dominios contemplados en la Taxonomía II de NANDA que son propios en el momento de intervenir la familia como son: Promoción de la Salud, Rol/Relaciones, Afrontamiento/Tolerancia al Estrés y Principios Vitales.

Los hallazgos obtenidos en la investigación tienen relación con lo establecido por Raile A y Marriner T, en la teoría de Betty Neuman, puesto que para esta teorista, "los elementos estresantes son estímulos productores de tensión que se generan dentro de los límites del sistema cliente y que dan lugar a un resultado que puede ser positivo o negativo ${ }^{(17)}$, las cuales pueden ser producto de tres fuerzas que intervienen en la salud de la persona, familias y como unidades, estas son: fuerzas 
intrapersonales, es decir aquellos factores propios del individuo, las fuerzas interpersonales tienen que ver entre uno o más individuos y están relacionadas con el rol y las fuerzas extrapersonales son las que son productos de factores externos del individuo, como aspectos económicos ${ }^{(17)}$.

Además, de acuerdo a lo enunciado por Lima J, Lima M y Sáez A, para que el profesional en enfermería realice el proceso de atención de enfermería en la familia, es necesario que identifique la "descripción de la familia, estudio de la dinámica familiar, mecanismos de defensa familiar y agentes estresantes" (6) para de esta forma poder abordar aquellos problemas que se presenten en este núcleo familiar. En donde la descripción de la familia, hace referencia a cómo está integrada la familia, las etapas del ciclo vital de la misma, las creencias, valores, hábitos, aspectos étnicos, religión, nivel socio-económico, problemas de salud, entre otros; la dinámica familiar, contempla aquellos aspectos en donde se analiza la comunicación, normas, roles, relación y adaptación del grupo familiar; los mecanismos de defensa familiar consisten en aquellas habilidades, actitudes que van relacionadas con el estado de salud, los conocimientos, toma de decisiones, recursos, experiencias, apoyos sociales, afrontamiento que tienen los integrantes de la familia ante situaciones o problemas y finalmente los agentes estresantes, en donde intervienen los acontecimientos vitales y las etapas del ciclo vital de la familia ${ }^{(6)}$.

Por medio de esta investigación, se pudo demostrar que la realización de instrumentos de valoración familiar enfocados en el lenguaje estandarizado son de gran importancia, puesto que permiten orientar al profesional en enfermería a realizar el/los cuidado/s, de acuerdo a las necesidades encontradas en la población a intervenir. Es indispensable, hacer uso de los referentes disciplinares que contribuyen significativamente en la profesión de enfermería, para de esta manera realizar una adecuada intervención basada en referentes teóricos propias de la profesión.

\section{CONCLUSIONES}

Con el Modelo de Lawshe Modificado, se encontró consenso general entre los jueces evaluadores en la validación de contenido.

Se estableció la fiabilidad del instrumento, por medio del Coeficiente Alpha de Cronbach, el cual arrojó un resultado de 0.847.

La correlación no paramétrica Rho de Spearman estableció que los cuatro dominios usados en el Instrumento de Valoración Familiar (Promoción de la Salud, Rol/Relaciones, Afrontamiento/Tolerancia al Estrés y Principios Vitales), establecidos en la NANDA 2012-2014, tienen relación entre sí, logrando un valor de $r$ mayor de 0.5 (cero punto cinco) lo cual indica una buena correlación entre los ítems contemplados en el instrumento y un valor de $\mathrm{p}$ de 0 (cero), es decir tienen un nivel de significancia $<0.05$.

\section{REFERENCIAS}

1. COLOMBIA. CONSEJO SUPERIOR DE LA JUDICATURA, SALA ADMINISTRATIVA. Constitución Política de Colombia 1991: Título II, capítulo 2 de los derechos sociales, económicos y culturales [en la internet]; 2010. [citado 2014 Marzo 28]. Disponible en: http://www.ramajudicial.gov.co/documents/10228/1547471/CONSTITUCIONInteriores.pdf/8b580886-d987-4668-a7a8-53f026f0f3a 
2. Villalobos Vásquez B. Efectividad de la funcionalidad familiar en las familias con hijos en preescolar, matriculados en el Colegio San Rafael Bogotá 2008. [Tesis Maestría]. Bogotá: Facultad de Enfermería, Universidad Nacional; 2009.

3. Gómez E, Haz A. Familias multiproblemáticas y en riesgo social: características e intervención. PSYKHE. 2007; 16 (2):43-54.

4. Fundación Universitaria Juan N Corpas. Guías: Modelos de atención en Salud Familiar con enfoque de riesgo. Departamento de Medicina Comunitaria [en la internet]; 2011. [citado 2014 Febrero 12]; Disponible en: http://www.juanncorpas.edu.co/uploads/media/CartillaDefinitivaTalleresPoliciaDic9de2 011.pdf

5. Lima Rodriguez J, Lima Serrano M, Jimenez Picón N, Domínguez Sánchez I. Consistencia interna y validez de un cuestionario para medir la autopercepción del estado de salud familiar. Rev. Esp. de Salud Pública. 2012; 5: 509-521.

6. Lima, J., Lima, M. y Sáez, A. Intervenciones enfermeras orientadas a la familia. Enfermería clínica. 2009; 19 (5): 280-283.

7. Louro Bernal Isabel. Matriz de salud del grupo familiar: un recurso para el diagnóstico de la situación de salud de la familia. Rev Cubana Med Gen Integr [revista en la Internet]. 2004. [citado 2014 Octubre 10] ; 20(3): Disponible en: http://scielo.sld.cu/scielo.php?script=sci_arttext\&pid=S086421252004000300006\&lng=es

8. Tristán López A, Molgado Ramos D. Tablas de validez de contenido (TVC). México: Instituto de Evaluación e Ingeniería Avanzada, S. C.; 2007.

9. Tristán López A. Modificación al modelo de Lawshe para el dictamen cuantitativo de la validez de contenido de un instrumento objetivo. Avances en medición. 2008; 6:37-48.

10.Sánchez R, Echeverry J. Validación de Escalas de Medición en Salud. Rev.Salud pública. 2004; 6 (3): 302-318.

11. Diaz L, Muñoz A, De Vargas D. Confiabilidad y validez del cuestionario de espiritualidad de Parsian y Dunning en versión española. Rev Latino-Am Enfermagem. 2012; 20 (3): 3-7.

12. Polit D., Hungler B. Estadística inferencial. En: Investigación científica en ciencias e la salud. México: McGraw-Hill: 2000; 405-434.

13. Giraldo Osorio A., Toro Rosero M., Macías Ladino A., Valencia C., Palacio Rodriguez S. La promoción de la salud como estrategia para el fomento de estilos de vida saludables. Hacia la promoción de la salud. 2010; 51 (1):128-143.

14. Suarez Cuba M. El médico familiar y la atención a la familia. Revista Paceña de Medicina Familiar. 2006; 3 (4): 95-100.

15. Spag J., Poblete F. Promoción de la Salud. 2007. [recuperado 2014 marzo 21]. Disponible en: http://medicinafamiliar.uc.cl/html/promocion_salud.html

16. Gómez, E., Kotlierenco, M. Resiliencia familiar: un enfoque de investigación e intervención con familias multiproblemáticas. Revista de psicología. 2010; 19(2): 103132.

17. Raile Alligood M., Marriner Tomey A. Modelos y teorías en enfermería. Barcelona, España: Elsevier: 2011.

18. Herrera E., Alzate M. Creencias y prácticas en el cuidado de la salud. Avances en Enfermería. 2008; XXVI (1): 112-123.

19. Dominguez Nariño C, Camargo Figueroa F, Flórez García J, Domínguez Torres L, Luna Figueroa D. Validación de un formato para algunos diagnósticos de enfermería de interés en la familia. Revista Cubana de Enfermería. 2013; 29 (2): 102-120. 
Recibido: 18 de mayo 2015;

Aceptado: 4 de agosto 2015

ISSN 1695-6141

(c) COPYRIGHT Servicio de Publicaciones - Universidad de Murcia 